\title{
July 2015 Pulmonary Case of the Month: A Crazy Case
}

\author{
Lewis J. Wesselius, MD \\ Department of Pulmonary Medicine \\ Mayo Clinic Arizona \\ Scottsdale, AZ
}

History of Present IIIness

A 23-year-old woman presented in 2008 at outside institution with dyspnea and diffuse pulmonary infiltrates. She required intubation. After a surgical lung biopsy, she was transferred to the Mayo Clinic Hospital for further care.

\section{Past Medical History}

She has had a history of progressive dyspnea for several months, otherwise negative.

\section{Physical Examination}

Vital signs are stable. SpO2 94\% on $\mathrm{FiO} 2$ of 0.4 . She is intubated and there is a chest tube in her right chest. Otherwise the physical examination is unremarkable.

\section{Radiography}

A thoracic CT scan was performed (Figure 1).

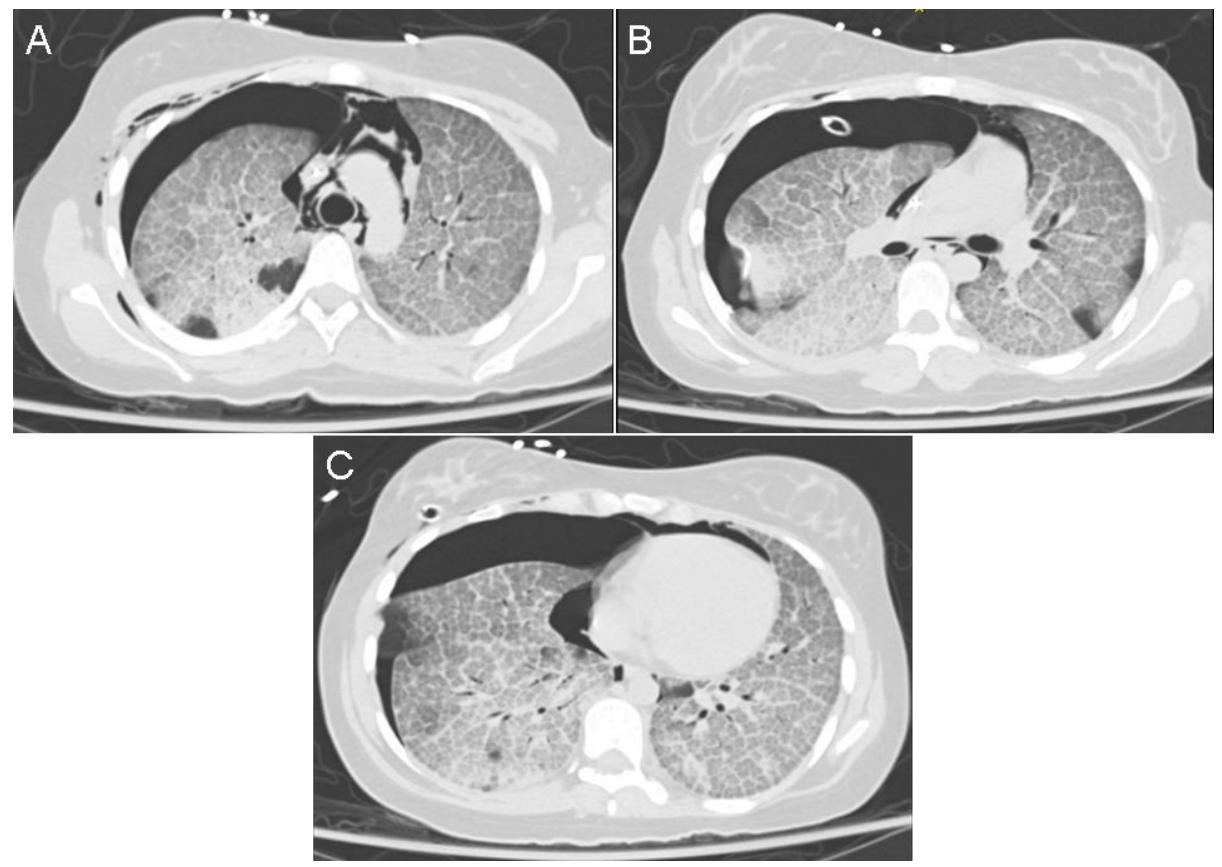

Figure 1. Representative images from the thoracic CT in lung windows. 
Which of the following are present on the thoracic CT scan?

1. Diffuse ground-glass opacities

2. Interlobular septal thickening and intralobular reticular thickening

3. Right-sided pneumothorax

4. 1 and 3

5. All of the above 


\section{Correct! \\ 5. All of the above}

There is a residual pneumothorax from the open lung biopsy, a chest tube present, diffuse ground glass opacities and inter- and intralobular septal thickening (Figure 2).

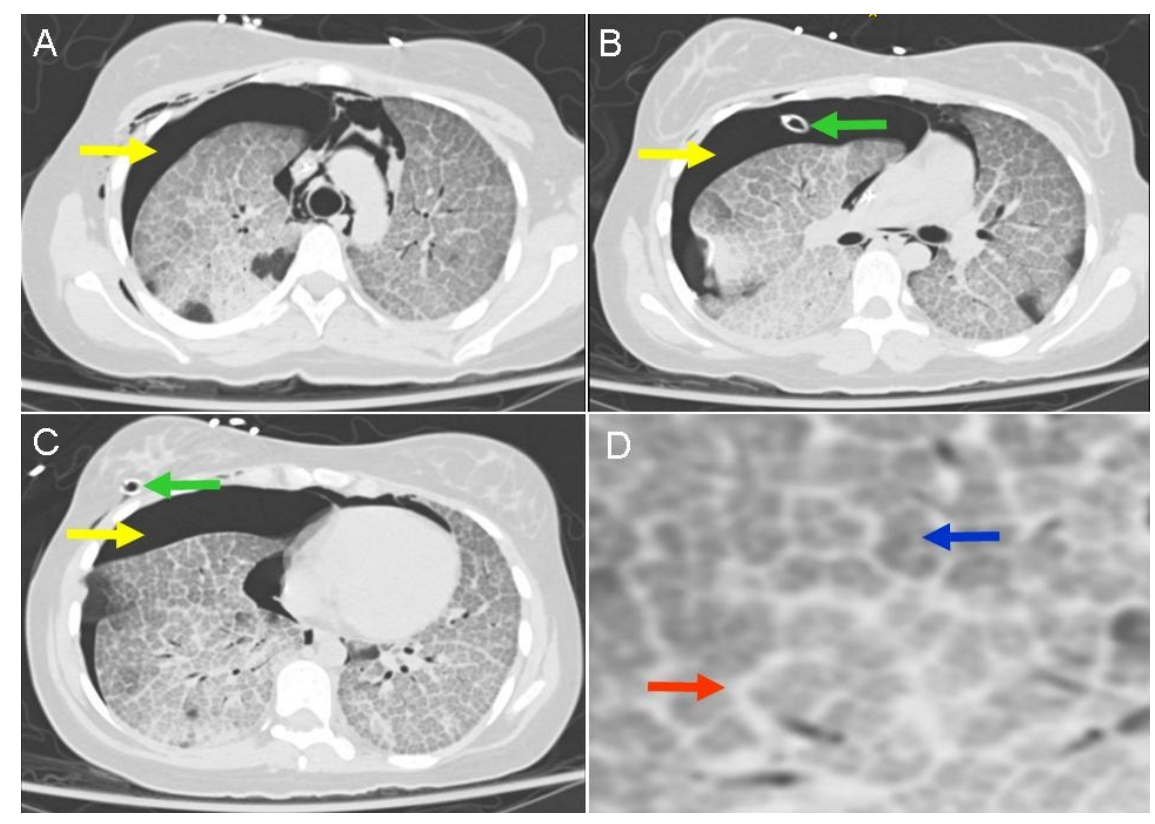

Figure 2. Panels A-C: Thoracic CT scan showing pneumothorax (yellow arrows) and chest tube (green arrows). Panel D: enlargement of the right lung showing interlobular septal thickening (red arrow) and intralobular septal thickening (blue arrow).

The lung biopsy performed at the outside hospital was requested (Figure 3).

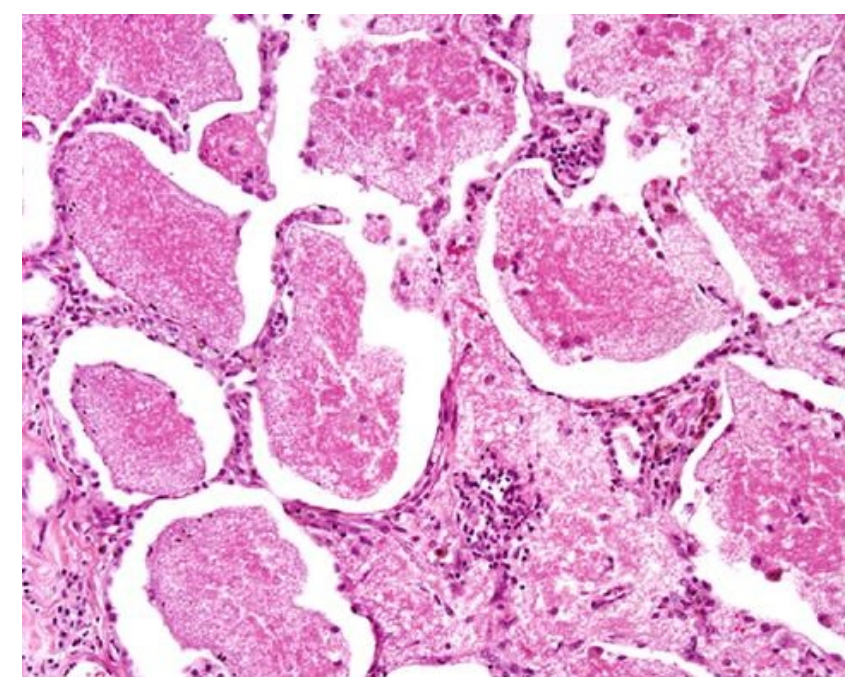

Figure 3. High power view of the open lung biopsy from the outside material showing positive periodic acid-Schiff (PAS) material in the alveoli. 
What is the diagnosis?

1. Alveolar proteinosis

2. Idiopathic pulmonary fibrosis

3. Mycobacterial pneumonia

4. Pneumocystis jiroveci pneumonia

5. Sarcoidosis 


\section{Correct! \\ 1. Alveolar proteinosis}

The clinical course, the radiologic pattern and the open lung biopsy are all compatible with alveolar proteinosis. Both the "crazy paving" pattern seen on the thoracic CT scan and the positive PAS material are nonspecific but in this clinical context are diagnostic of alveolar proteinosis.

Which of the following would be appropriate therapy in this case?

1. Begin granulocyte macrophage-colony stimulating factor (GM-CSF)

2. Radiation to the whole lung

3. Whole lung lavage

4. 1 and 3

5. All of the above 


\section{Correct!}

\section{1 and 3}

There are 3 types of alveolar proteinosis (1):

1. Congenital

2. Secondary (allogeneic bone marrow transplant, hematologic malignancy, infections (Pneumocystis), acute toxic inhalations [silica, aluminum])

3. Acquired (autoimmune).

About $90 \%$ of the cases of alveolar proteinosis are acquired. This occurs when due antiGM-CSF antibodies are formed which result in impairment of the alveolar macrophage clearance of surfactant. The surfactant accumulates clogging the alveoli resulting in the dyspnea and the positive PAS staining. Our patient's serum was positive for antibodies to GM-CSF.

The treatment of choice for severely ill patients is whole lung lavage (1). She was also begun on GM-CSF.

After whole lung lavage she was weaned off ventilator. After discharge pulmonary function tests (PFTs) showed a FVC of $80 \%$ of predicted, a TLC of $79 \%$ of predicted, and a DLco of $74 \%$ of predicted. A thoracic CT scan marked improvement in her "crazy paving" (Figure 4).

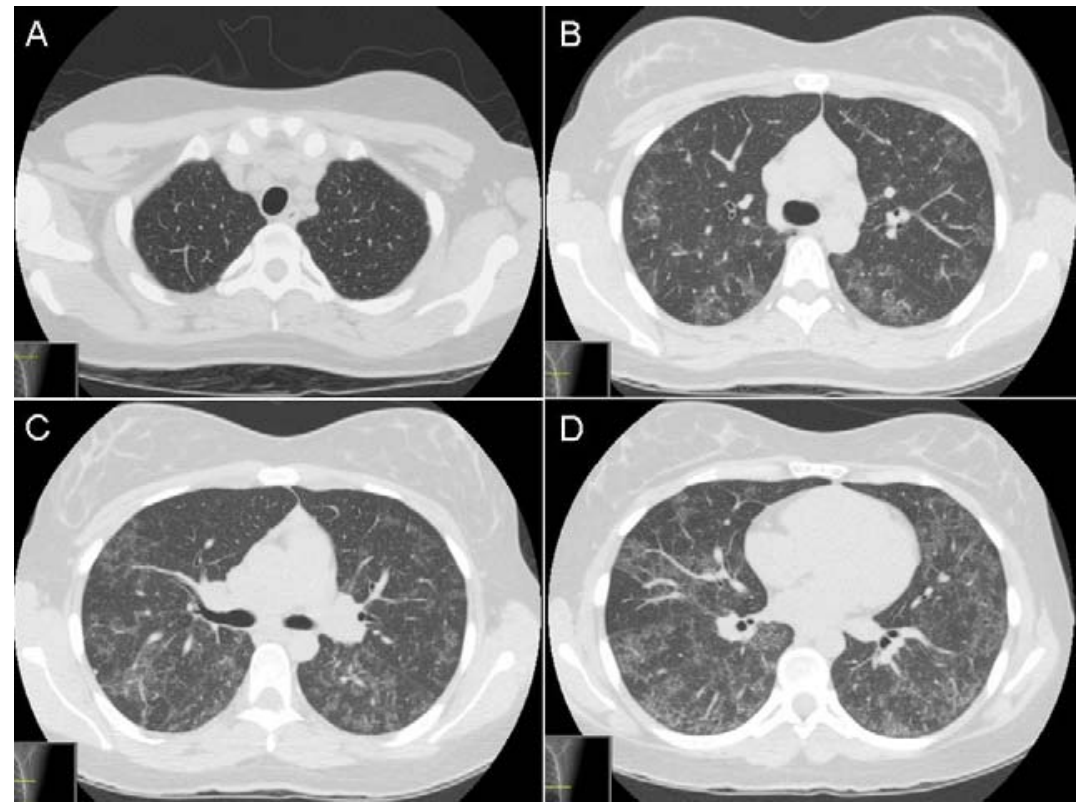

Figure 4. Representative images from the repeat thoracic CT scan showing improvement in the "crazy paving" compared to Figure 1.

By November, 2009 she had completed 4 months of GM-CSF and was feeling well, not requiring oxygen. She was subsequently lost to follow-up. In February, 2014 she was referred by her obstetrician because she was now pregnant with increasing dyspnea. 
Repeat PFTs showed her FVC was $56 \%$ of predicted and her DLco was $42 \%$ of predicted. Chest $\mathrm{x}$-ray showed diffuse consolidation.

Which of the following are indicated at this time?

1. Lung transplant

2. Rituximab

3. Therapeutic abortion

4. Whole lung lavage

5. None of the above 


\section{Correct! \\ 5. None of the above}

There are multiple treatment options available for alveolar proteinosis including (2):

1. Whole lung lavage

2. GM-CSF: systemic or inhaled

3. Rituximab (1 series of 10 patients)

4. Plasmapharesis (case reports)

5. Lung transplant (relapses reported)

6. Spontaneous remissions occur in up to $25 \%$ (no reported association with pregnancy)

None of these seemed appropriate in this situation and she was followed while on supplemental oxygen. She had a successful delivery in August, 2014 of a healthy child. She felt her breathing had improved after delivery and GM-CSF was restarted. Repeat PFTs in October, 2014 showed her FVC was $66 \%$ of predicted and her DLco 50\% predicted although her chest x-ray continued to show diffuse consolidation (Figure 5).

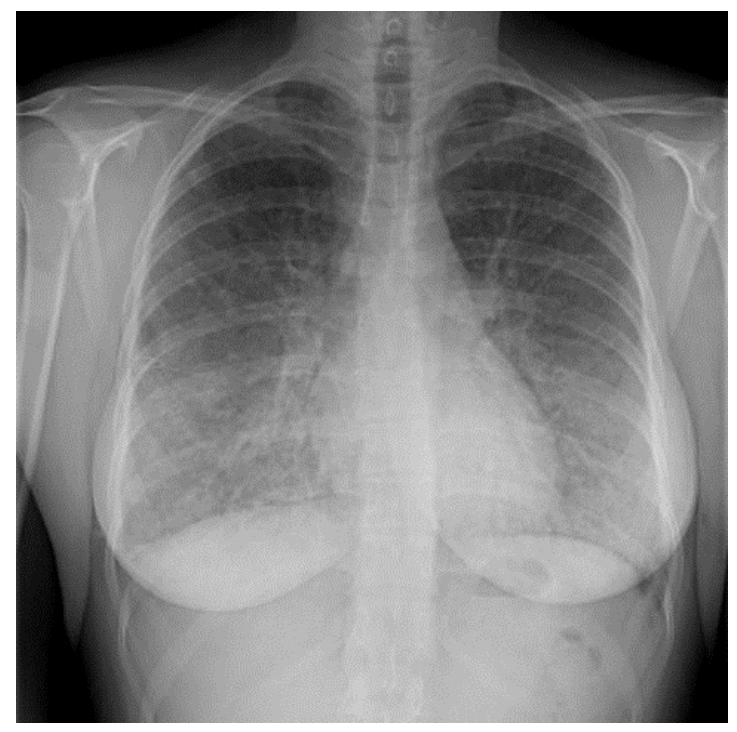

Figure 5. Repeat chest x-ray showing diffuse consolidation.

She continued to improve and in April, 2015 her FVC was $80 \%$ of predicted and her DLco was $70 \%$ of predicted.

\section{References}

1. Presneill JJ, Nakata K, Inoue $\mathrm{Y}$, Seymour JF. Pulmonary alveolar proteinosis. Clin Chest Med. 2004;25(3):593-613. [CrossRef] [PubMed]

2. Luisetti M, Kadija Z, Mariani F, Rodi G, Campo I, Trapnell BC. Therapy options in pulmonary alveolar proteinosis. Ther Adv Respir Dis. 2010;4(4):239-48. [CrossRef] [PubMed] 